DESY 99-011

ISSN $0418-9833$

quant-ph/9902017

February 1999

\title{
PATH INTEGRAL SOLUTIONS FOR DEFORMED PÖSCHL-TELLER-LIKE AND CONDITIONALLY SOLVABLE POTENTIALS
}

\author{
Christian Grosche \\ II. Institut für Theoretische Physik \\ Universität Hamburg, Luruper Chaussee 149 \\ 22761 Hamburg, Germany
}

\begin{abstract}
I discuss in this paper the behaviour of the solutions of the so-called $q$-hyperbolic potentials, i.e. Pöschl-Teller-like and conditionally solvable potentials, in terms of the path integral formalism. The differences in comparison to the usual Pöschl-Teller-like potentials are investigated, including the discrete energy spectra and the bound state wave-functions.
\end{abstract}




\section{Introduction.}

In this paper I want to discuss some specific generalisations of Pöschl-Teller related potentials. They are based on a $q$-deformation of the usual hyperbolic potentials, and are denoted by (we assume without loss of generality $q>0$ )

$$
\sinh _{q} x=\frac{1}{2}\left(\mathrm{e}^{x}-q \mathrm{e}^{-x}\right), \quad \cosh _{q} x=\frac{1}{2}\left(\mathrm{e}^{x}+q \mathrm{e}^{-x}\right) .
$$

Consequently we define

$$
\tanh _{q} x=\frac{\sinh _{q} x}{\cosh _{q} x}, \quad \tanh _{q} x=\frac{\cosh _{q} x}{\sinh _{q} x} .
$$

Note the relation $\cosh _{q}^{2} x-\sinh _{q}^{2} x=q$, which has the consequence that almost all relations known from the usual hyperbolic functions must be modified. In analogy to the usual hyperbolic functions we have on the one hand

$$
\frac{\mathrm{d}}{\mathrm{d} x} \cosh _{q} x=\sinh _{q} x, \quad \frac{\mathrm{d}}{\mathrm{d} x} \sinh _{q} x=\cosh _{q} x .
$$

However, on the other hand we obtain

$$
\frac{\mathrm{d}}{\mathrm{d} x} \tanh _{q} x=\frac{q}{\cosh _{q}^{2} x}, \quad \frac{\mathrm{d}}{\mathrm{d} x} \operatorname{coth}_{q} x=-\frac{q}{\sinh _{q}^{2} x} .
$$

These potentials also belong the class of shape invariant potentials as derived from supersymmetric quantum mechanics [19, 9], and have been first introduced by Arai [1], and furthermore discussed by Lévai [23] and Lemieux and Bose [22] in the context of general solutions of the hypergeometric equation. Recently, these potentials have been also discussed by Eğrifes et al. [6]. The introduction of the parameter $q$ may serve as an additional parameter in describing inter-atomic interactions. The usual potentials based on the Pöschl-Teller type provide only at most two parameters. We can therefore investigate whether it is possible to introduce the additional parameter $q$ to modify a sample of known potentials which are related to the (modified) Pöschl-Teller potential in order to change the energy-level feature of the potentials.

The simplest system of such a deformed potential with bound state solutions based on the $q$-deformed hyperbolic functions has the form $(x \in \mathbb{R})$

$$
V_{1}(x)=-\frac{\hbar^{2}}{2 m} \frac{\nu^{2}-1 / 4}{\cosh _{q}^{2} x}=-\frac{\hbar^{2}}{2 m} \frac{\nu^{2}-1 / 4}{\frac{1}{2}\left(\mathrm{e}^{x}+q \mathrm{e}^{-x}\right)^{2}} .
$$

Extracting a factor $\sqrt{q}$ we obtain

$$
V_{1}(x)=-\frac{\hbar^{2}}{2 m q} \frac{\nu^{2}-1 / 4}{\frac{1}{2}\left(\mathrm{e}^{-\ln \sqrt{q}+x}+\mathrm{e}^{\ln \sqrt{q}-x}\right)^{2}} .
$$

If we define $y=x-\ln \sqrt{q} \in \mathbb{R}$ we obtain

$$
V_{1}(y)=\frac{\left.V_{1}(x)\right|_{q=1}}{q}=-\frac{\hbar^{2}}{2 m q} \frac{\nu^{2}-1 / 4}{\cosh ^{2} y},
$$

and the only effect is a scaling of the potential. The corresponding Lagrangian is changed in the following way

$$
\mathcal{L}=\frac{m}{2} \dot{x}^{2}+\frac{\hbar^{2}}{2 m} \frac{\nu^{2}-1 / 4}{\cosh _{q}^{2} x} \quad \rightarrow \quad \frac{m}{2} \dot{y}^{2}+\frac{\hbar^{2}}{2 m q} \frac{\nu^{2}-1 / 4}{\cosh ^{2} y}
$$


A more complicate version of this potential is the full modified Pöschl-Teller potential, now in the form of $q$-deformed hyperbolic functions, i.e. $(x>\ln \sqrt{q})$

$$
\begin{aligned}
V_{2}(x) & =\frac{\hbar^{2}}{2 m}\left(\frac{\eta^{2}-1 / 4}{\sinh _{q}^{2} x}-\frac{\nu^{2}-1 / 4}{\cosh _{q}^{2} x}\right) \\
& =\frac{\hbar^{2}}{2 m}\left(\frac{\eta^{2}-1 / 4}{\frac{1}{4}\left(\mathrm{e}^{x}-q \mathrm{e}^{-x}\right)^{2}}-\frac{\nu^{2}-1 / 4}{\frac{1}{4}\left(\mathrm{e}^{x}+q \mathrm{e}^{-x}\right)^{2}}\right) .
\end{aligned}
$$

Performing the transformation $y=x-\ln \sqrt{q}>0$ yields

$$
V_{2}(y)=\frac{\left.V_{2}(x)\right|_{q=1}}{q}=\frac{\hbar^{2}}{2 m q}\left(\frac{\eta^{2}-1 / 4}{\sinh ^{2} y}-\frac{\nu^{2}-1 / 4}{\cosh ^{2} y}\right),
$$

and the only effect is again a scaling of the potential.

For another type of these kind of potentials (Manning-Rosen potential) which is related to the Coulomb potential in hyperbolic geometry we define

$$
\begin{aligned}
V_{3}(x) & =-\alpha \operatorname{coth}_{q} x+\frac{\hbar^{2}}{2 m} \frac{\lambda^{2}-1 / 4}{\sinh _{q}^{2} x} \\
& =-\alpha \frac{\mathrm{e}^{x}+q \mathrm{e}^{-x}}{\mathrm{e}^{x}-q \mathrm{e}^{-x}}+\frac{\hbar^{2}}{2 m q} \frac{\lambda^{2}-1 / 4}{\left(\mathrm{e}^{-\ln \sqrt{q}+x}-\mathrm{e}^{\ln \sqrt{q}-x}\right)^{2}} .
\end{aligned}
$$

Performing the same transformation as before we get $(x>\ln \sqrt{q})$

$$
V_{3}(y)=-\alpha \operatorname{coth} y+\frac{\hbar^{2}}{2 m q} \frac{\lambda^{2}-1 / 4}{\sinh ^{2} y},
$$

and for the Lagrangian, respectively

$$
\begin{aligned}
\mathcal{L} & =\frac{m}{2} \dot{x}^{2}+\alpha \operatorname{coth} q-\frac{\hbar^{2}}{2 m} \frac{\lambda^{2}-1 / 4}{\sinh _{q}^{2} x} \\
& \rightarrow \frac{m}{2} \dot{y}^{2}+\alpha \operatorname{coth} y-\frac{\hbar^{2}}{2 m q} \frac{\lambda^{2}-1 / 4}{\sinh ^{2} y}
\end{aligned}
$$

and only the "radial" potential strength is now modified. Here we can expect a significant modification of the spectral properties due to the additional parameter $q$.

A fourth kind of potential (Rosen-Morse potential) is defined by $(x \in \mathbb{R})$

$$
\begin{aligned}
V_{4}(x) & =\beta \tanh _{q} x-\frac{\hbar^{2}}{2 m} \frac{\lambda^{2}-1 / 4}{\cosh _{q}^{2} x} \\
& =\beta \frac{\mathrm{e}^{x}-q \mathrm{e}^{-x}}{\mathrm{e}^{x}+q \mathrm{e}^{-x}}-\frac{\hbar^{2}}{2 m} \frac{\lambda^{2}-1 / 4}{\left(\mathrm{e}^{-\ln \sqrt{q}+x}+\mathrm{e}^{\ln \sqrt{q}-x}\right)^{2}} .
\end{aligned}
$$

Performing the same transformation as before we get

$$
V_{4}(y)=\beta \tanh y+\frac{\hbar^{2}}{2 m q} \frac{\lambda^{2}-1 / 4}{\cosh ^{2} y},
$$


and for the Lagrangian, respectively

$$
\begin{aligned}
\mathcal{L} & =\frac{m}{2} \dot{x}^{2}+\beta \tanh _{q} x+\frac{\hbar^{2}}{2 m} \frac{\lambda^{2}-1 / 4}{\cosh _{q}^{2} x} \\
& \rightarrow \frac{m}{2} \dot{y}^{2}-\beta \tanh y+\frac{\hbar^{2}}{2 m q} \frac{\lambda^{2}-1 / 4}{\cosh ^{2} y},
\end{aligned}
$$

and again only the "radial" potential strength is modified.

Consequently, the $q$-deformed hyperbolic Scarf potential [12] is defined by $(x>\ln \sqrt{q})$

$$
\begin{aligned}
V_{5}(x) & =V_{0}+V_{1} \operatorname{coth}_{q}^{2} x+V_{2} \frac{\operatorname{coth}_{q} x}{\sinh _{q} x} \\
& \rightarrow V_{0}+V_{1} \operatorname{coth} y+\frac{V_{2}}{\sqrt{q}} \frac{\operatorname{coth} y}{\sinh y} .
\end{aligned}
$$

The $q$-deformed hyperbolic barrier potential [12] is defined by $(x \in \mathbb{R})$

$$
\begin{aligned}
V_{6}(x) & =V_{0}+V_{1} \frac{\tanh _{q} x}{\cosh _{q} x}+V_{2} \tanh _{q}^{2} x \\
& \rightarrow V_{0}+\frac{V_{1}}{\sqrt{q}} \frac{\tanh y}{\cosh y}+V_{2} \tanh ^{2} y .
\end{aligned}
$$

There are four kinds of conditionally solvable potentials [14, 15]. We introduce $(x \in \mathbb{R}, r>$ $\ln \sqrt{q}, y=x-\ln \sqrt{q}, z=r-\ln \sqrt{q})$

$$
\begin{aligned}
V_{7}(x) & =\frac{\hbar^{2}}{2 m}\left(-\frac{A \mathrm{e}^{-x}}{\sqrt{1+q \mathrm{e}^{-2 x}}}+\frac{B}{1+q \mathrm{e}^{-2 x}}+\frac{C}{\left(1+q \mathrm{e}^{-2 x}\right)^{2}}\right) \\
& \rightarrow \frac{\hbar^{2}}{2 m}\left(-\frac{(A / \sqrt{q}) \mathrm{e}^{-y / 2}}{\sqrt{2 \cosh y}}+\frac{B \mathrm{e}^{y}}{2 \cosh y}+\frac{C \mathrm{e}^{2 y}}{4 \cosh ^{2} y}\right) \\
V_{7}^{\prime}(x) & =\frac{\hbar^{2}}{2 m}\left(-\frac{A}{\sqrt{1+q \mathrm{e}^{-2 x}}}+\frac{B}{1+q \mathrm{e}^{-2 x}}+\frac{C}{\left(1+q \mathrm{e}^{-2 x}\right)^{2}}\right) \\
& \rightarrow \frac{\hbar^{2}}{2 m}\left(-\frac{A \mathrm{e}^{y / 2}}{\sqrt{2 \cosh y}}\right)+\frac{B \mathrm{e}^{y}}{2 \cosh y}+\frac{C \mathrm{e}^{2 y}}{4 \cosh ^{2} y}, \\
V_{8}(r) & =\frac{\hbar^{2}}{2 m}\left(f+1-\frac{f-3 / 4}{1-q \mathrm{e}^{-2 r}}+\frac{h_{1} \mathrm{e}^{-r}}{\sqrt{1-q \mathrm{e}^{-2 r}}+\frac{C}{\left(1-q \mathrm{e}^{-2 r}\right)^{2}}}\right) \\
& \rightarrow \frac{\hbar^{2}}{2 m}\left(f+1-\frac{(f-3 / 4) \mathrm{e}^{z}}{2 \sinh z}+\frac{(h / \sqrt{q}) e^{-z / 2}}{\sqrt{2 \sinh z}}+\frac{C \mathrm{e}^{2 z}}{4 \sinh ^{2} z}\right), \\
V_{8}^{\prime}(r) & =\frac{\hbar^{2}}{2 m}\left(f+1-\frac{f-3 / 4}{1-q \mathrm{e}^{-2 r}}+\frac{h_{1}}{\sqrt{1-q \mathrm{e}^{-2 r}}}+\frac{C}{\left(1-q \mathrm{e}^{-2 r}\right)^{2}}\right) \\
\rightarrow & \frac{\hbar^{2}}{2 m}\left(f+1-\frac{(f-3 / 4) \mathrm{e}^{z}}{2 \sinh z}+\frac{h_{1} e^{z / 2}}{\sqrt{2 \sinh z}}+\frac{C \mathrm{e}^{2 z}}{4 \sinh ^{2} z}\right) .
\end{aligned}
$$

Here I have adopted the notation from [14, 15]. The potentials $V_{7}, V_{7}^{\prime}$ may be called "deformed modified Rosen-Morse potential II and I", respectively, and the potentials $V_{8}, V_{8}^{\prime}$ deformed 
Manning-Rosen potentials II and I", respectively. These potentials are also called "conditionally solvable", c.f. [5, 14, 15, 25] and references therein, because exact solutions can only be found if the parameter $C$ takes on the values $C=3 / 4$. In the potentials $V_{7}$ and $V_{8}$ we indeed can change the behaviour of the feature of the potential, whereas in the potentials $V_{7}^{\prime}, V_{8}^{\prime}$ there is only a scaling due to a simple shift of coordinates.

\section{Path Integral Solution}

\subsection{The Potential $V_{1}$.}

We start with the potential $V_{1}$. According to [2, 17, 21] the solution is given in terms of the corresponding Green function $G$ of the Feynman kernel. The path integral solution of the potential $V_{1}$ is simple, because we can directly apply the path integral solution for the symmetric modified Pöschl-Teller potential [21]. Explicitly we have:

$$
\begin{aligned}
& \int_{x\left(t^{\prime}\right)=x^{\prime}}^{x\left(t^{\prime \prime}\right)=x^{\prime \prime}} \mathcal{D} x(t) \exp \left[\frac{\mathrm{i}}{\hbar} \int_{t^{\prime}}^{t^{\prime \prime}}\left(\frac{m}{2} \dot{x}^{2}+\frac{\hbar^{2}}{2 m} \frac{\lambda^{2}-\frac{1}{4}}{\cosh _{q}^{2} x}\right) \mathrm{d} t\right] \\
& =\int_{y\left(t^{\prime}\right)=y^{\prime}}^{y\left(t^{\prime \prime}\right)=y^{\prime \prime}} \mathcal{D} y(t) \exp \left[\frac{\mathrm{i}}{\hbar} \int_{t^{\prime}}^{t^{\prime \prime}}\left(\frac{m}{2} \dot{y}^{2}+\frac{\hbar^{2}}{2 m} \frac{\tilde{\lambda}^{2}-\frac{1}{4}}{\cosh ^{2} y}\right) \mathrm{d} t\right]=\int_{\mathbb{R}} \frac{\mathrm{d} E}{2 \pi \mathrm{i}} G^{\left(V_{1}\right)}\left(x^{\prime \prime}, x^{\prime} ; E\right), \\
& G^{\left(V_{1}\right)}\left(x^{\prime \prime}, x^{\prime} ; E\right)=\frac{m}{\hbar^{2}} \Gamma\left(\frac{1}{\hbar} \sqrt{-2 m E}-\tilde{\lambda}+\frac{1}{2}\right) \Gamma\left(\frac{1}{\hbar} \sqrt{-2 m E}+\tilde{\lambda}+\frac{1}{2}\right) \\
& \quad \times P_{\tilde{\lambda}-1 / 2}^{-\sqrt{-2 m E} / \hbar}\left(\tanh y_{<}\right) P_{\tilde{\lambda}-1 / 2}^{-\sqrt{-2 m E} / \hbar}\left(-\tanh y_{>}\right),
\end{aligned}
$$

where I have set $\tilde{\lambda}^{2}=\left(\lambda^{2}-1 / 4\right) / q+1 / 4$; note the relation

$$
\tanh y=\frac{q^{-1 / 2} \mathrm{e}^{x}-q^{1 / 2} \mathrm{e}^{-x}}{q^{-1 / 2} \mathrm{e}^{x}+q^{1 / 2} \mathrm{e}^{-x}}=\tanh _{q} x .
$$

The $P_{\nu}^{\mu}(z)$ are Legendre functions. The bound states are given by

$$
\Psi^{\left(V_{1}\right)}(x)=\left(\frac{n-\tilde{\lambda}-\frac{1}{2}}{q} \frac{\Gamma(2 \tilde{\lambda}-n)}{n !}\right)^{1 / 2} P_{\tilde{\lambda}-1 / 2}^{n-\tilde{\lambda}+\frac{1}{2}}\left(\tanh _{q} x\right)
$$

and the energy spectrum is given by

$$
E_{n}^{\left(V_{1}\right)}=-\frac{\hbar^{2}}{2 m}\left(n-\tilde{\lambda}+\frac{1}{2}\right)^{2}
$$

where $n=0,1, \ldots, N_{\max }<\left[\tilde{\lambda}-\frac{1}{2}\right]$, and $[x]$ denotes the integer values of $x \in \mathbb{R}$. The continuous solutions we do not state, c.f. [17, 21]. We observe that the principal effect consists in a change in the parameter $\lambda \rightarrow \tilde{\lambda}$. Depending whether $0<q<1$ or $q>1$ there is an increasing respectively decreasing of the energy levels and the number of energy levels in comparison to the original $1 / \cosh ^{2} x$ problem. 


\subsection{The Potential $V_{2}$.}

Next, we consider the potential $V_{2}$, the deformed Pöschl-Teller potential. We express the solution in terms of a path integral, and again the Green function can be stated in closed form as known from the literature [12, 21] $(x>\ln \sqrt{q})$

$$
\begin{aligned}
& \int_{x\left(t^{\prime}\right)=x^{\prime}}^{x\left(t^{\prime \prime}\right)=x^{\prime \prime}} \mathcal{D} x(t) \exp \left\{\frac{\mathrm{i}}{\hbar} \int_{t^{\prime}}^{t^{\prime \prime}}\left[\frac{m}{2} \dot{x}^{2}-\frac{\hbar^{2}}{2 m}\left(\frac{\eta^{2}-\frac{1}{4}}{\sinh _{q}^{2} x}-\frac{\nu^{2}-\frac{1}{4}}{\cosh _{q}^{2} x}\right)\right] \mathrm{d} t\right\} \\
& =\int_{y\left(t^{\prime}\right)=y^{\prime}}^{y\left(t^{\prime \prime}\right)=y^{\prime \prime}} \mathcal{D} y(t) \exp \left\{\frac{\mathrm{i}}{\hbar} \int_{t^{\prime}}^{t^{\prime \prime}}\left[\frac{m}{2} \dot{y}^{2}-\frac{\hbar^{2}}{2 m q}\left(\frac{\eta^{2}-\frac{1}{4}}{\sinh ^{2} y}-\frac{\nu^{2}-\frac{1}{4}}{\cosh ^{2} y}\right)\right] \mathrm{d} t\right\}=\int_{\mathbb{R}} \frac{\mathrm{d} E}{2 \pi \mathrm{i}} G^{\left(V_{2}\right)}\left(x^{\prime \prime}, x^{\prime} ; E\right) \text {, } \\
& G^{\left(V_{2}\right)}\left(x^{\prime \prime}, x^{\prime} ; E\right)=\frac{m}{\hbar^{2}} \frac{\Gamma\left(m_{1}-L_{\nu}\right) \Gamma\left(L_{\nu}+m_{1}+1\right)}{\Gamma\left(m_{1}+m_{2}+1\right) \Gamma\left(m_{1}-m_{2}+1\right)} \\
& \times\left(q \cosh _{q} x^{\prime} \cosh _{q} x^{\prime \prime}\right)^{-\left(m_{1}-m_{2}\right)}\left(\tanh _{q} x^{\prime} \tanh _{q} x^{\prime \prime}\right)^{m_{1}+m_{2}+1 / 2} \\
& \times_{2} F_{1}\left(-L_{\nu}+m_{1}, L_{\nu}+m_{1}+1 ; m_{1}-m_{2}+1 ; q \cosh _{q}^{-2} x_{<}\right) \\
& \times_{2} F_{1}\left(-L_{\nu}+m_{1}, L_{\nu}+m_{1}+1 ; m_{1}+m_{2}+1 ; \tanh _{q}^{2} x_{>}\right) \text {, } \\
& {\left[m_{1,2}=\frac{1}{2}(\tilde{\eta} \pm \sqrt{-2 m E} / \hbar), L_{\nu}=\frac{1}{2}(\tilde{\nu}-1), \tilde{\eta}^{2}=\left(\eta^{2}-1 / 4\right) / q+1 / 4, \tilde{\nu}^{2}=\left(\nu^{2}-1 / 4\right) / q+1 / 4\right] .} \\
& { }_{2} F_{1}(a, b ; c ; z) \text { is the hypergeometric function. The bound states are } \\
& \begin{array}{l}
\Psi_{n}^{(\eta, \nu)}(x)=N_{n}^{(\eta, \nu)}\left(q^{-1 / 2} \sinh _{q} r\right)^{\eta+1 / 2}\left(q^{-1 / 2} \cosh _{q} x\right)^{n-\nu+1 / 2}{ }_{2} F_{1}\left(-n, \tilde{\nu}-n ; 1+\tilde{\eta} ; \tanh _{q}^{2} x\right) \\
N_{n}^{(\eta, \nu)}=\frac{1}{\Gamma(1+\tilde{\eta})}\left[\frac{2(\tilde{\nu}-\tilde{\eta}-2 n-1) \Gamma(n+1+\tilde{\eta}) \Gamma(\nu-n)}{\Gamma(\nu-\tilde{\eta}-n) n !}\right]^{1 / 2} \\
E_{n}=-\frac{\hbar^{2}}{2 m}(2 n+\tilde{\eta}-\tilde{\nu}-1)^{2}, \quad n=0,1, \ldots, N_{\max }<\left[\frac{1}{2}(\tilde{\nu}-\tilde{\eta}-1)\right]
\end{array}
\end{aligned}
$$

Again, we omit the continuous states.

\subsection{The Potential $V_{3}$.}

The Manning-Rosen Potential with deformed hyperbolic functions by considering a space-time transformation in the path integral (Duru-Kleinert transformation) [20]. We have $(x>\ln \sqrt{q})$

$$
\begin{aligned}
& \int_{x\left(t^{\prime}\right)=x^{\prime}}^{x\left(t^{\prime \prime}\right)=x^{\prime \prime}} \mathcal{D} x(t) \exp \left[\frac{\mathrm{i}}{\hbar} \int_{t^{\prime}}^{t^{\prime \prime}}\left(\frac{m}{2} \dot{x}^{2}+\alpha \operatorname{coth} q-\frac{\hbar^{2}}{2 m} \frac{\lambda^{2}-1 / 4}{\sinh _{q}^{2} x}\right) \mathrm{d} t\right] \\
& =\int_{y\left(t^{\prime}\right)=y^{\prime}}^{y\left(t^{\prime \prime}\right)=y^{\prime \prime}} \mathcal{D} y(t) \exp \left[\frac{\mathrm{i}}{\hbar} \int_{t^{\prime}}^{t^{\prime \prime}}\left(\frac{m}{2} \dot{y}^{2}+\alpha \operatorname{coth} y-\frac{\hbar^{2}}{2 m q} \frac{\lambda^{2}-1 / 4}{\sinh ^{2} y}\right) \mathrm{d} t\right]=\int_{\mathbb{R}} \frac{\mathrm{d} E}{2 \pi \mathrm{i}} G^{\left(V_{3}\right)}\left(x^{\prime \prime}, x^{\prime} ; E\right), \\
& G^{\left(V_{3}\right)}\left(x^{\prime \prime}, x^{\prime} ; E\right)=\frac{m}{\hbar^{2}} \frac{\Gamma\left(m_{1}-L_{E}\right) \Gamma\left(L_{E}+m_{1}+1\right)}{\Gamma\left(m_{1}+m_{2}+1\right) \Gamma\left(m_{1}-m_{2}+1\right)}
\end{aligned}
$$




$$
\begin{aligned}
& \times\left(\frac{2}{\operatorname{coth}_{q} x^{\prime}+1} \cdot \frac{2}{\operatorname{coth}_{q} x^{\prime \prime}+1}\right)^{\left(m_{1}+m_{2}+1\right) / 2}\left(\frac{\operatorname{coth}_{q} x^{\prime}-1}{\operatorname{coth}_{q} x^{\prime}+1} \cdot \frac{\operatorname{coth}_{q} x^{\prime \prime}-1}{\operatorname{coth}_{q} x^{\prime \prime}+1}\right)^{\left(m_{1}-m_{2}\right) / 2} \\
& \times{ }_{2} F_{1}\left(-L_{E}+m_{1}, L_{E}+m_{1}+1 ; m_{1}-m_{2}+1 ; \frac{\operatorname{coth}_{q} x_{>}-1}{\operatorname{coth}_{q} x_{>}+1}\right) \\
& \times{ }_{2} F_{1}\left(-L_{E}+m_{1}, L_{E}+m_{1}+1 ; m_{1}+m_{2}+1 ; \frac{2}{\operatorname{coth}_{q} x_{<}+1}\right),
\end{aligned}
$$

where $L_{E}=-\frac{1}{2}+\sqrt{2 m(\alpha-E)} / 2$, and $m_{1,2}=\frac{1}{2}\left(2 \tilde{\lambda} \pm \frac{1}{\hbar} \sqrt{-2 m(\alpha+E)}\right)$, and $\tilde{\lambda}$ defined as in $V_{1}$. The relevant coordinate- and time-transformations to obtain a path integral formulation in terms of the modified Pöschl-Teller potential have the form [10, 21] (this will not be repeated here once more, $r>0$ )

$$
\frac{1}{2}(1-\operatorname{coth} y)=-\frac{1}{\sinh ^{2} r}, \quad \mathrm{~d} t=\tanh ^{2} r \mathrm{~d} s .
$$

The wave functions and the energy spectrum of the bound states read $\left(0,1, \ldots \leq N_{\max }<\right.$ $\left[\sqrt{m \alpha / 2} / \hbar-\frac{1}{2}(s+1)\right], s=2 \tilde{\lambda}, k_{2}=(1+s) / 2, k_{1}=\left(1+(s+2 n+1) / 2+2 m \alpha / \hbar^{2}(s+2 n+1)\right) / 2$, note $\left.n+\frac{1}{2}-k_{1}<0\right)$ :

$$
\begin{aligned}
\Psi_{n}(x) & =\left[\left(1+\frac{4 m|\alpha|}{\hbar(s+2 n+1)^{2}}\right) \frac{\left(2 k_{1}-2 n-s-2\right) n ! \Gamma\left(2 k_{1}-n-1\right)}{\Gamma(n+s+1) \Gamma\left(2 k_{1}-s-n-1\right)}\right]^{1 / 2} \\
\times & \left(1-q \mathrm{e}^{-2 x}\right)^{(s+1) / 2} \mathrm{e}^{-(2 x-\ln \sqrt{q})\left(k_{1}-s / 2-n-1\right)} P_{n}^{\left(2 k_{1}-2 n-s-2, s\right)}\left(1-2 q \mathrm{e}^{-2 x}\right),
\end{aligned}
$$

and the energy spectrum has the form

$$
E_{n}=-\frac{\hbar^{2}(s+2 n+1)^{2}}{8 m}-\frac{2 m \alpha^{2}}{\hbar^{2}(s+2 n+1)^{2}} .
$$

The $P_{n}^{(\alpha, \beta)}$ are Jacobi polynomials. The number of bound states is determined by $N_{\max }$, which depends on $\alpha$ and $s$. Decreasing $s$ for fixed $\alpha$ is archived by $0<q<1$.

\subsection{The Potential $V_{4}$.}

For the Rosen-Morse potential in $q$-deformed hyperbolic functions we obtain $(x \in \mathbb{R})$

$$
\begin{aligned}
& \int_{x\left(t^{\prime}\right)=x^{\prime}}^{x\left(t^{\prime \prime}\right)=x^{\prime \prime}} \mathcal{D} x(t) \exp \left[\frac{\mathrm{i}}{\hbar} \int_{t^{\prime}}^{t^{\prime \prime}}\left(\frac{m}{2} \dot{x}^{2}-\beta \tanh _{q} x+\frac{\hbar^{2}}{2 m} \frac{\lambda^{2}-1 / 4}{\cosh _{q}^{2} x}\right) \mathrm{d} t\right] \\
& =\int_{y\left(t^{\prime}\right)=y^{\prime}}^{y\left(t^{\prime \prime}\right)=y^{\prime \prime}} \mathcal{D} y(t) \exp \left[\frac{\mathrm{i}}{\hbar} \int_{t^{\prime}}^{t^{\prime \prime}}\left(\frac{m}{2} \dot{x}^{2}-\beta \tanh y+\frac{\hbar^{2}}{2 m q} \frac{\lambda^{2}-1 / 4}{\cosh ^{2} y}\right) \mathrm{d} t\right]=\int_{\mathbb{R}} \frac{\mathrm{d} E}{2 \pi \mathrm{i}} G^{\left(V_{4}\right)}\left(x^{\prime \prime}, x^{\prime} ; E\right), \\
& G^{\left(V_{4}\right)}\left(x^{\prime \prime}, x^{\prime} ; E\right)=\frac{m}{\hbar^{2}} \frac{\Gamma\left(m_{1}-L_{B}\right) \Gamma\left(L_{B}+m_{1}+1\right)}{\Gamma\left(m_{1}+m_{2}+1\right) \Gamma\left(m_{1}-m_{2}+1\right)} \\
& \quad \times\left(\frac{1-\tanh _{q} x^{\prime}}{2} \cdot \frac{1-\tanh _{q} x^{\prime \prime}}{2}\right)^{\frac{m_{1}-m_{2}}{2}}\left(\frac{1+\tanh _{q} x^{\prime}}{2} \cdot \frac{1+\tanh _{q} x^{\prime \prime}}{2}\right)^{\frac{m_{1}+m_{2}}{2}}
\end{aligned}
$$




$$
\begin{aligned}
& \times_{2} F_{1}\left(-L_{B}+m_{1}, L_{B}+m_{1}+1 ; m_{1}+m_{2}+1 ; \frac{1+\tanh _{q} x_{>}}{2}\right) \\
& \times_{2} F_{1}\left(-L_{B}+m_{1}, L_{B}+m_{1}+1 ; m_{1}-m_{2}+1 ; \frac{1-\tanh _{q} x_{<}}{2}\right),
\end{aligned}
$$

$L_{B}=-\frac{1}{2}+2 \tilde{\lambda}, m_{1,2}=\sqrt{m / 2}(\sqrt{-\beta-E} \pm \sqrt{\beta-E}) / \hbar$. The relevant coordinate- and timetransformations to obtain a path integral formulation in terms of the modified Pöschl-Teller potential have the form [10, 21] (this will not be repeated here once more, $r>0$ )

$$
\frac{1}{2}(1+\tanh y)=\tanh ^{2} r, \quad \mathrm{~d} t=\operatorname{coth}^{2} r \mathrm{~d} s .
$$

The wave functions and the energy spectrum are given by $\left(s \equiv 2 \tilde{\lambda} ; 0, \ldots, n \leq N_{\max }<\left[\frac{1}{2}(s-\right.\right.$ $\left.1)-\sqrt{m|\beta| / 2} / \hbar], k_{1}=\frac{1}{2}(1+s), k_{2}=\frac{1}{2}\left(1+\frac{1}{2}(s-2 n-1)-\frac{2 m A}{\hbar(s-2 n-1)}\right)>\frac{1}{2}\right):$

$$
\begin{aligned}
\Psi_{n}= & {\left[\left(1-\frac{4 m|\beta|}{\hbar(s-2 n-1)^{2}}\right) \frac{\left(s-2 k_{2}-2 n\right) n ! \Gamma(s-n)}{\Gamma\left(s+1-n-2 k_{2}\right) \Gamma\left(2 k_{2}+n\right)}\right]^{1 / 2} 2^{n+(1-s) / 2} } \\
& \times\left(1-\tanh _{q} x\right)^{\frac{1}{2} s-k_{2}-n}\left(1+\tanh _{q} x\right)^{k_{2}-\frac{1}{2}} P_{n}^{\left(s-2 k_{2}-2 n, 2 k_{2}-1\right)}\left(\tanh _{q} x\right), \\
E_{n}= & -\left[\frac{\hbar^{2}(s-2 n-1)^{2}}{8 m}+\frac{2 m \beta^{2}}{\hbar^{2}(s-2 n-1)^{2}}\right] .
\end{aligned}
$$

The number of bound states is determined by $N_{\max }$, which depends on $\alpha$ and $s$. Increasing $s$ for fixed $\beta$ is archived by $q>1$.

\subsection{The Potential $V_{5}$.}

For the $q$-deformed hyperbolic Scarf Potential we obtain $(x>\ln \sqrt{q}$, here the coordinate transformation consists just in $x \rightarrow x / 2$ )

$$
\begin{aligned}
& \int_{x\left(t^{\prime}\right)=x^{\prime}}^{x\left(t^{\prime \prime}\right)=x^{\prime \prime}} \mathcal{D} x(t) \exp \left\{\frac{\mathrm{i}}{\hbar} \int_{t^{\prime}}^{t^{\prime \prime}}\left[\frac{m}{2} \dot{x}^{2}-\frac{\hbar^{2}}{2 m}\left(V_{0}+V_{1} \operatorname{coth}_{q}^{2} x+V_{2} \frac{\operatorname{coth}_{q} x}{\sinh _{q} x}\right)\right] \mathrm{d} t\right\} \\
& =\int_{y\left(t^{\prime}\right)=y^{\prime}}^{y\left(t^{\prime \prime}\right)=y^{\prime \prime}} \mathcal{D} y(t) \exp \left\{\frac{\mathrm{i}}{\hbar} \int_{t^{\prime}}^{t^{\prime \prime}}\left[\frac{m}{2} \dot{y}^{2}-\frac{\hbar^{2}}{2 m}\left(V_{0}+V_{1} \operatorname{coth}^{2} y+\frac{V_{2}}{\sqrt{q}} \frac{\operatorname{coth} y}{\sinh y}\right)\right] \mathrm{d} t\right\} \\
& =\int_{\mathbb{R}} \frac{\mathrm{d} E}{2 \pi \mathrm{i}} G^{\left(V_{5}\right)}\left(x^{\prime \prime}, x^{\prime} ; E\right), \\
& G^{\left(V_{5}\right)}\left(x^{\prime \prime}, x^{\prime} ; E\right)=\frac{2 m}{\hbar^{2}} \frac{\Gamma\left(m_{1}-L_{\nu}\right) \Gamma\left(L_{\nu}+m_{1}+1\right)}{\Gamma\left(m_{1}+m_{2}+1\right) \Gamma\left(m_{1}-m_{2}+1\right)} \\
& \quad \times\left(q^{-1 / 2} \cosh _{q} \frac{x^{\prime}}{2} \cosh _{q} \frac{x^{\prime \prime}}{2}\right)^{-\left(m_{1}-m_{2}\right)}\left(\tanh _{q} \frac{x^{\prime}}{2} \tanh _{q} \frac{x^{\prime \prime}}{2}\right)^{m_{1}+m_{2}+1 / 2} \\
& \quad \times{ }_{2} F_{1}\left(-L_{\nu}+m_{1}, L_{\nu}+m_{1}+1 ; m_{1}-m_{2}+1 ; q^{1 / 2} \cosh _{q}^{-2} \frac{x_{<}}{2}\right) \\
& \quad \times{ }_{2} F_{1}\left(-L_{\nu}+m_{1}, L_{\nu}+m_{1}+1 ; m_{1}+m_{2}+1 ; \tanh _{q}^{2} \frac{x_{>}}{2}\right),
\end{aligned}
$$

with $m_{1,2}=\eta / 2 \pm \sqrt{V_{0}+V_{1}-2 m E / \hbar^{2}}$, where $\eta=\sqrt{V_{1}+V_{2} / \sqrt{q}+1 / 4}, \nu=\sqrt{V_{1}-V_{2} / \sqrt{q}+1 / 4}$, and $L_{\nu}=\frac{1}{2}(\nu-1)$. The bound-state wave-functions and the energy spectrum are given by

$$
\Psi_{n}(x)=\left[\frac{\left(2 k_{1}-2 k_{2}-2 n-1\right) n ! \Gamma\left(2 k_{1}-n-1\right)}{2 \Gamma\left(2 k_{2}+n\right) \Gamma\left(2 k_{1}-2 k_{2}-n\right)}\right]^{1 / 2}\left(q^{-1 / 4} \sinh _{q} \frac{x}{2}\right)^{2 k_{2}-1 / 2}
$$




$$
\begin{aligned}
& \times\left(q^{-1 / 4} \cosh _{q} \frac{x}{2}\right)^{2 n-2 k_{1}+3 / 2} P_{n}^{\left[2 k_{2}-1,2\left(k_{1}-k_{2}-n\right)-1\right]}\left(\frac{2 q^{1 / 2}}{\cosh _{q}^{2} \frac{x}{2}}-1\right), \\
E_{n}= & \left.\frac{\hbar^{2}}{2 m}\left(V_{0}+V_{1}\right)-\frac{\hbar^{2}}{2 m}\left[\left(k_{1}-k_{2}-n\right)-\frac{1}{2}\right)\right]^{2} .
\end{aligned}
$$

Here we denote $n=0,1, \ldots, N_{\max }<k_{1}-k_{2}-1 / 2, k_{1}=\frac{1}{2}\left(1+\sqrt{V_{1}-V_{2} / \sqrt{q}+1 / 4}\right), k_{2}=$ $\frac{1}{2}\left(1+\sqrt{V_{1}+V_{2} / \sqrt{q}+1 / 4}\right)$, and $\kappa=k_{1}-k_{2}-n$. In order that bound states can exist, it is required that $V_{2}<0$.

\subsection{The Potential $V_{6}$.}

The $q$-deformed barrier potential is treated in a similar way. We obtain $(x \in \mathbb{R}$, together with the coordinate transformation $(1+\mathrm{i} \sinh x) / 2=\cosh ^{2} r$ with in order to obtain a modified Pöschl-Teller potential in the new coordinate $r>0$, which will be also not be repeated again 12])

$$
\begin{aligned}
& \int_{x\left(t^{\prime}\right)=x^{\prime}}^{x\left(t^{\prime \prime}\right)=x^{\prime \prime}} \mathcal{D} x(t) \exp \left\{\frac{\mathrm{i}}{\hbar} \int_{t^{\prime}}^{t^{\prime \prime}}\left[\frac{m}{2} \dot{x}^{2}-\frac{\hbar^{2}}{2 m}\left(V_{0}+V_{1} \frac{\tanh _{q} x}{\cosh _{q} x}+V_{2} \tanh _{q}^{2} x\right)\right] \mathrm{d} t\right\} \\
& =\int_{y\left(t^{\prime}\right)=y^{\prime}}^{y\left(t^{\prime \prime}\right)=y^{\prime \prime}} \mathcal{D} y(t) \exp \left\{\frac{\mathrm{i}}{\hbar} \int_{t^{\prime}}^{t^{\prime \prime}}\left[\frac{m}{2} \dot{x}^{2}-\frac{\hbar^{2}}{2 m}\left(V_{0}+\frac{V_{1}}{\sqrt{q}} \frac{\tanh y}{\cosh y}+V_{2} \tanh ^{2} y\right)\right] \mathrm{d} t\right\} \\
& =\int_{\mathbb{R}} \frac{\mathrm{d} E}{2 \pi \mathrm{i}} G^{\left(V_{6}\right)}\left(x^{\prime \prime}, x^{\prime} ; E\right), \\
& G^{\left(V_{6}\right)}\left(x^{\prime \prime}, x^{\prime} ; E\right)=\frac{m}{\hbar^{2}} \frac{\Gamma\left(m_{1}-L_{\nu}\right) \Gamma\left(L_{\nu}+m_{1}+1\right)}{\Gamma\left(m_{1}+m_{2}+1\right) \Gamma\left(m_{1}-m_{2}+1\right)} \\
& \quad \times\left(q^{-1} \cosh _{q} r^{\prime} \cosh _{q} r^{\prime \prime}\right)^{-\left(m_{1}-m_{2}\right)}\left(\tanh _{q} r^{\prime} \tanh _{q} r^{\prime \prime}\right)^{m_{1}+m_{2}+\frac{1}{2}} \\
& \quad \times{ }_{2} F_{1}\left(-L_{\nu}+m_{1}, L_{\nu}+m_{1}+1 ; m_{1}-m_{2}+1 ; q \cosh _{q}^{-2} x_{<}\right) \\
& \quad \times{ }_{2} F_{1}\left(-L_{\nu}+m_{1}, L_{\nu}+m_{1}+1 ; m_{1}+m_{2}+1 ; \tanh _{q}^{2} x_{>}\right),
\end{aligned}
$$

with $\eta=\sqrt{V_{2}-\mathrm{i} V_{1} / \sqrt{q}+1 / 4}, \nu=\sqrt{V_{2}+\mathrm{i} V_{1} / \sqrt{q}+1 / 4}, L_{\nu}=\frac{1}{2}(\nu-1)$, and $m_{1,2}=\eta / 2 \pm$ $\sqrt{V_{0}+V_{2} / \sqrt{q}-2 m E / \hbar^{2}}$. Furthermore we have $k_{1}=\frac{1}{2} \sqrt{V_{2} / \sqrt{q}-\mathrm{i} V_{1}+\frac{1}{4}} \equiv \frac{1}{2}(1+\lambda), k_{2}=$ $\frac{1}{2}\left(1-\lambda^{*}\right)$, with the wave-functions $\left(\lambda_{R, I}=(\Re, \Im)(\lambda), n=0,1, \ldots, N_{\max }<\left[\lambda_{R}-\frac{1}{2}\right]\right)$

$$
\begin{aligned}
\Psi_{n}(x) & =\left[\frac{\left(2 \lambda_{R}-2 n-1\right) n ! \Gamma(\lambda-n)}{2 \Gamma\left(2 \lambda_{R}-n\right) \Gamma\left(n+1-\lambda^{*}\right)}\right]^{1 / 2} \\
& \times\left(\frac{1+\mathrm{i} q^{-1 / 2} \sinh _{q} x}{2}\right)^{\frac{1}{2}\left(\frac{1}{2}-\lambda\right)}\left(\frac{1-\mathrm{i} q^{-1 / 2} \sinh _{q} x}{2}\right)^{\frac{1}{2}\left(\frac{1}{2}-\lambda^{*}\right)} P_{n}^{\left(-\lambda^{*},-\lambda\right)}\left(\mathrm{i} q^{-1 / 2} \sinh _{q} x\right)
\end{aligned}
$$

with the energy spectrum

$$
E_{n}=\frac{\hbar^{2}}{2 m}\left(V_{0}+V_{2}\right)-\frac{\hbar^{2}}{2 m}\left\{n+\frac{1}{2}-\sqrt{\frac{1}{2}\left[\sqrt{\left.\left(\frac{1}{4}+V_{2}\right)^{2}+\frac{V_{1}^{2}}{q}+\frac{1}{4}+V_{2}\right]}\right.}\right\}^{2},
$$

The energy spectrum is modified by the varying $q$ in the $V_{1}^{2}$-term. 


\subsection{The Potential $V_{7}$.}

The solution of the path integral for the potential $V_{7}$ is related to the solution of the (deformed) hyperbolic Scarf potential [14]. We have for the path integral formulation

$$
\begin{aligned}
& \int_{x\left(t^{\prime}\right)=x^{\prime}}^{x\left(t^{\prime \prime}\right)=x^{\prime \prime}} \mathcal{D} x(t) \exp \left\{\frac{\mathrm{i}}{\hbar} \int_{t^{\prime}}^{t^{\prime \prime}}\left[\frac{m}{2} \dot{x}^{2}-\frac{\hbar^{2}}{2 m}\left(-\frac{A \mathrm{e}^{-x}}{\sqrt{1+q \mathrm{e}^{-2 x}}}+\frac{B}{1+q \mathrm{e}^{-2 x}}+\frac{C}{\left(1+q \mathrm{e}^{-2 x}\right)^{2}}\right)\right] \mathrm{d} t\right\} \\
& =\int_{y\left(t^{\prime}\right)=y^{\prime}}^{y\left(t^{\prime \prime}\right)=y^{\prime \prime}} \mathcal{D} y(t) \exp \left\{\frac{\mathrm{i}}{\hbar} \int_{t^{\prime}}^{t^{\prime \prime}}\left[\frac{m}{2} \dot{x}^{2}-\frac{\hbar^{2}}{2 m}\left(-\frac{(A / \sqrt{q}) \mathrm{e}^{-y / 2}}{\sqrt{2 \cosh y}}+\frac{B \mathrm{e}^{y}}{2 \cosh y}+\frac{C \mathrm{e}^{2 y}}{4 \cosh ^{2} y}\right)\right] \mathrm{d} t\right\} \\
& =\int_{\mathbb{R}} \frac{\mathrm{d} E}{2 \pi \mathrm{i}} G^{\left(V_{7}\right)}\left(x^{\prime \prime}, x^{\prime} ; E\right),
\end{aligned}
$$

The details of its solution are not repeated here again, c.f. also $V_{5}$. The quantization condition is found to read $(\tilde{B}=B / \sqrt{q})$

$$
\sqrt{A-E_{n}-\frac{3 \hbar^{2}}{8 m}}=\frac{1}{2}\left(\sqrt{\tilde{B}-E_{n}}-\sqrt{-\tilde{B}-E_{n}}\right)-\frac{\hbar}{\sqrt{2 m}}\left(n+\frac{1}{2}\right),
$$

This give after some algebra a cubic equation in $\left(-E_{n}\right)\left(\lambda=A+C+\tilde{n}^{2}, C=-3 \hbar^{2} / 8 m, \tilde{n}=\right.$ $\left.\hbar\left(n+\frac{1}{2}\right) / \sqrt{2 m}\right)$

$$
\begin{aligned}
4 \tilde{n}^{2}( & \left.-E_{n}\right)^{3}+\left[12 \tilde{n}^{2}\left(\tilde{n}^{2}+\lambda\right)-\lambda^{2}\right]\left(-E_{n}\right)^{2} \\
& +\left[16 \tilde{n}^{2} \lambda(A+C+\lambda)-2\left(\lambda+4 \tilde{n}^{2}\right)\left(\lambda^{2}+\frac{\tilde{B}^{2}}{4}+4 \tilde{n}^{2}(A+C)\right)\right]\left(-E_{n}\right) \\
& +\left[16 \tilde{n}^{2} \lambda^{2}(A+C)-\left(\lambda^{2}+\frac{B^{2}}{4}+4 \tilde{n}^{2}(A+C)\right)^{2}\right]=0 .
\end{aligned}
$$

From the Green function of the hyperbolic Scarf-like potential we derive the Green function for the potential $V_{7}$

$$
\begin{aligned}
G^{\left(V_{7}\right)} & \left(x^{\prime \prime}, x^{\prime} ; E\right)=\left(\operatorname{coth} u^{\prime} \operatorname{coth} u^{\prime \prime}\right)^{1 / 2} \frac{2 m}{\hbar^{2}} \frac{\Gamma\left(m_{1}-L_{\nu}\right) \Gamma\left(L_{\nu}+m_{1}+1\right)}{\Gamma\left(m_{1}+m_{2}+1\right) \Gamma\left(m_{1}-m_{2}+1\right)} \\
& \times\left(\cosh u^{\prime} \cosh u^{\prime \prime}\right)^{-\left(m_{1}-m_{2}\right)}\left(\tanh u^{\prime} \tanh u^{\prime \prime}\right)^{m_{1}+m_{2}+\frac{1}{2}} \\
& \times{ }_{2} F_{1}\left(-L_{\nu}+m_{1}, L_{\nu}+m_{1}+1 ; m_{1}-m_{2}+1 ; \frac{1}{\cosh ^{2} u_{<}}\right) \\
& \times{ }_{2} F_{1}\left(-L_{\nu}+m_{1}, L_{\nu}+m_{1}+1 ; m_{1}+m_{2}+1 ; \tanh ^{2} u_{>}\right)
\end{aligned}
$$

with $\sinh u=\mathrm{e}^{y}=\mathrm{e}^{x-\ln \sqrt{q}}, m_{1,2}=\eta / 2 \pm \sqrt{V_{0}+V_{1}-8 m E / \hbar^{2}}$, where $\eta=\sqrt{V_{1}+V_{2}+1 / 4}, L_{\nu}=$ $\frac{1}{2}(\nu-1)$ and $\nu=\sqrt{V_{1}-V_{2}+1 / 4}$, together with the identification $V_{0}=2 m A / \hbar^{2}-\frac{1}{2}, V_{1}=$ $-\left(2 m E / \hbar^{2}+\frac{1}{4}\right), V_{2}=-2 m \tilde{B} / \hbar^{2}$. The poles of the Green function determine the energy-spectrum, and the corresponding residua give the wave-functions expansions. We obtain $\left(k_{1}=\frac{1}{2}(1+\nu), k_{2}=\right.$ $\left.\frac{1}{2}(1+\eta), \eta=\sqrt{-2 m\left(E_{n}+\tilde{B}\right)} / \hbar, \nu=\sqrt{2 m\left(\tilde{B}-E_{n}\right)} / \hbar\right)$

$$
E_{n}=\sqrt[3]{\sqrt{D}+\frac{Q}{2}}-\sqrt[3]{\sqrt{D}-\frac{Q}{2}}+\frac{R}{3}
$$




$$
\begin{aligned}
& D=\left(\frac{P}{3}\right)^{3}+\left(\frac{Q}{2}\right)^{2}, \quad P=\frac{3 S-R^{2}}{3}, \quad Q=\frac{2 R^{3}}{27}-\frac{R S}{3}+T \\
& R=\frac{12 \tilde{n}^{2}\left(\tilde{n}^{2}+\lambda\right)-\lambda^{2}}{4 \tilde{n}^{2}}, \quad T=\frac{16 \tilde{n}^{2} \lambda^{2}(A+C)-\left[\lambda^{2}+\tilde{B}^{2} / 4+4 \tilde{n}^{2}(A+C)\right]^{2}}{4 \tilde{n}^{2}} \\
& S=\frac{8 \tilde{n}^{2} \lambda(A+C+\lambda)-\left(\lambda^{2}+4 \tilde{n}^{2}\right)\left(\lambda^{2}+\tilde{B}^{2} / 4+4 \tilde{n}^{2}(A+C)\right]}{2 \tilde{n}^{2}}
\end{aligned}
$$

We omit the details concerning the wave functions. Bound states exist if $A<0,0<\tilde{B}<|A|$, and the number $N_{\max }$ of bound states is found by requiring $\left|E_{n}\right|>\tilde{B}$.

\subsection{The Potential $V_{8}$.}

The path integral for the potential $V_{8}$ is related to the path integral for the hyperbolic barrier potential as discussed in [15]

$$
\begin{aligned}
& \int_{x\left(t^{\prime}\right)=x^{\prime}}^{x\left(t^{\prime \prime}\right)=x^{\prime \prime}} \mathcal{D} x(t) \exp \left\{\frac{\mathrm{i}}{\hbar} \int_{t^{\prime}}^{t^{\prime \prime}}\left[\frac{m}{2} \dot{x}^{2}-\frac{\hbar^{2}}{2 m}\left(f+1-\frac{f-3 / 4}{1-q \mathrm{e}^{-2 r}}+\frac{h_{1} \mathrm{e}^{-r}}{\sqrt{1-q \mathrm{e}^{-2 r}}}+\frac{C}{\left(1-q \mathrm{e}^{-2 r}\right)^{2}}\right)\right] \mathrm{d} t\right\} \\
& z\left(t^{\prime \prime}\right)=z^{\prime \prime} \\
& =\int_{z\left(t^{\prime}\right)=z^{\prime}} \mathcal{D} z(t) \\
& \times \exp \left\{\frac{\mathrm{i}}{\hbar} \int_{t^{\prime}}^{t^{\prime \prime}}\left[\frac{m}{2} \dot{x}^{2}-\frac{\hbar^{2}}{2 m}\left(f+1-\frac{(f-3 / 4) \mathrm{e}^{z}}{2 \sinh z}+\frac{(h / \sqrt{q}) e^{-z / 2}}{\sqrt{2 \sinh z}}+\frac{C \mathrm{e}^{2 z}}{4 \sinh ^{2} z}\right)\right] \mathrm{d} t\right\} \\
& =\int_{\mathbb{R}} \frac{\mathrm{d} E}{2 \pi \mathrm{i}} G^{\left(V_{8}\right)}\left(x^{\prime \prime}, x^{\prime} ; E\right),
\end{aligned}
$$

The details of its solution are not repeated here again, c.f. [15] Due to the fact that its solution is defined in the half-space $\mathbb{R}^{+}$, we must construct the corresponding Green function in terms of the Green function in the entire $\mathbb{R}$, a method described in 13. This has also been discussed in detail in [15] which is not repeated here. Hence we obtain $\left(\zeta(z)=\frac{1}{2}(1+\tanh z), z=r-\ln \sqrt{q}>0\right)$

$$
G^{\left(V_{8}\right)}(E)\left(x^{\prime \prime}, x^{\prime} ; E\right)=G\left(\zeta^{\prime \prime}, \zeta^{\prime} ; E\right)-\frac{G\left(\zeta^{\prime \prime}, \zeta(0) ; E\right) G\left(\zeta(0), \zeta^{\prime} ; E\right)}{G(\zeta(0), \zeta(0) ; E)},
$$

with the Green function $G(E)$ given by

$$
\begin{aligned}
& G\left(\zeta^{\prime \prime}, \zeta^{\prime} ; E\right)=\frac{m / \hbar^{2}}{\sqrt{\zeta\left(z^{\prime}\right) \zeta\left(z^{\prime \prime}\right)}} \frac{\Gamma\left(m_{1}-L_{\nu}\right) \Gamma\left(L_{\nu}+m_{1}+1\right)}{\Gamma\left(m_{1}+m_{2}+1\right) \Gamma\left(m_{1}-m_{2}+1\right)} \\
& \quad \times\left(\frac{1-\sqrt{\zeta\left(z^{\prime}\right)}}{2} \cdot \frac{1-\sqrt{\zeta\left(z^{\prime \prime}\right)}}{2}\right)^{\left(m_{1}-m_{2}\right) / 2}\left(\frac{1+\sqrt{\zeta\left(z^{\prime}\right)}}{2} \cdot \frac{1+\sqrt{\zeta\left(z^{\prime \prime}\right)}}{2}\right)^{\left(m_{1}+m_{2}+1 / 2\right) / 2} \\
& \quad \times{ }_{2} F_{1}\left(-L_{\nu}+M_{1}, L_{\nu}+m_{1}+1 ; m_{1}+m_{2}+1 ; \frac{1+\sqrt{\zeta_{>}(z)}}{2}\right) \\
& \quad \times{ }_{2} F_{1}\left(-L_{\nu}+M_{1}, L_{\nu}+m_{1}+1 ; m_{1}-m_{2}+1 ; \frac{1-\sqrt{\zeta_{<}(z)}}{2}\right) .
\end{aligned}
$$

Here I have used the abbreviations

$$
L_{\nu}=\frac{1}{2}\left(\sqrt{f+1+\mathrm{i} h_{1}-\frac{2 m}{\hbar^{2}} E}-1\right), \quad m_{1,2}=-\frac{1}{2} \sqrt{f+1-\mathrm{i} h_{1}-\frac{2 m}{\hbar^{2}}} E \pm \sqrt{\frac{1}{4}-f} .
$$


Note that the minus-sign in the first term in $m_{1,2}$ is due to the reality condition of the problem [15]. Bound states with energy $E_{n}$ are determined by the equation

$$
{ }_{2} F_{1}\left(-L_{\nu}\left(E_{n}\right)+m_{1}\left(E_{n}\right), L_{\nu}\left(E_{n}\right)+m_{1}\left(E_{n}\right)+1 ; m_{1}\left(E_{n}\right)+m_{2}\left(E_{n}\right)+1 ; \frac{1}{2}\right) .
$$

A more detailed numerical investigation of this transcendental equation involving the hypergeometric function is left to the reader.

\section{Summary and Discussion.}

The results of our investigation of the introduction of the $q$-deformed hyperbolic potentials show a combination of a shift of the coordinate origin of the potential combined with a scaling of the potential strength. In the cases of the potentials $V_{1}$ to $V_{6}$ the introduction of the parameter $q$ the energy levels and the wave functions were modified by a nonlinear, however simple way. In particular the energy levels could be easily derived from previous calculations. The cases of the potentials $V_{7}$ and $V_{8}$ were somewhat more difficult, which was due the fact that the energy levels are determined by a third-order equation and a transcendental equation, respectively. $q$ also entered the expressions nonlinearly. Taking into account the potentials $V_{7}^{\prime}$ and $V_{8}^{\prime}$ we would obtain energy spectra determined by a fourth-order equation and a transcendental equation, modified by a simple shift due to the coordinate translation.

Therefore these potentials can serve as modeling potentials where a finite potential trough is required for particle interaction in molecular, atomic or nuclear physics. This feature is in particular seen, if the potential is defined in the half-space $x>\ln \sqrt{q}$. Depending whether $0<q<1$ or $q>1$ the number of energy levels and the ground state energy can be increased or decreased, respectively. We see the convenience of the path integral formalism in the solutions of the deformed potential problems. We can easily use previous results, adapted accordingly to the present problems. In some way, the potentials constructed from $q$-deformed hyperbolic functions model in a very simple and convenient way coordinate translations. In the "radial" problems the introduction of the parameter $q$ forces the quantum motion to take place in the half-space $x>\ln \sqrt{q}$ and not in the half-space $x>0$. We therefore have introduced an impenetrable finite wall between the particle motion and the coordinate origin, which may be identified for instance with the center-of-mass location of a molecule. This feature alters the energy spectrum in a nonlinear way, in particular if in the $q=1$-case there is an integer quantum number $\lambda \equiv l \in \mathbb{N}$. However, this is a phenomenological feature and does not make new physics.

One should also keep in mind that the $q$-deformed hyperbolic potentials can be used to describe curvature in spaces of negative constant curvature, i.e., on hyperboloids (compare also [3] for the interrelation of a deformed algebra and the constant negative curvature in the model of the hyperbolic plane [11]). Let us consider the simplest hyperboloid

$$
u_{0}^{2}-u_{1}^{2}-u_{2}^{2}=R^{2}, \quad u_{0} \geq 0,
$$

which describes one sheet of the double-sheeted hyperboloid $\Lambda^{(2)}$. According to [16, 24] on $\Lambda^{(2)}$ there are nine coordinate systems which allow separation of variables in the Helmholtz, respectively Schrödinger equation. We consider the usual spherical system $(\tau \in \mathbb{R}, \varphi \in[0,2 \pi))$ :

$$
\left.\begin{array}{l}
u_{0}=R \cosh \tau, \\
u_{1}=R \sinh \tau \cos \varphi, \\
u_{2}=R \sinh \tau \sin \varphi,
\end{array}\right\} \quad \longrightarrow \quad\left\{\begin{array}{l}
u_{0}=\cosh _{q} \tau, \\
u_{1}=\sinh _{q} \tau \cos \varphi \\
u_{2}=\sinh _{q} \tau \sin \varphi
\end{array}\right.
$$


and we observe that with the identification $q=R^{2}$ the $q$-deformed spherical coordinate system is a possible separating coordinate system for $\Lambda^{(2)}$. Furthermore we obtain $-\left(\dot{u}_{0}^{2}-\dot{u}_{1}^{2}-\dot{u}_{2}^{2}\right)=$ $q \dot{\tau}^{2}+\sinh _{q}^{2} \tau \dot{\varphi}^{2}$. A calculation shows that the introduction of $q$ does not change the energy spectrum features for the free quantum motion on $\Lambda^{(2)}$ (just rescale $m \rightarrow m / q$ ). We can also consider the Higgs oscillator $V(\mathbf{u})=\left(m R^{2} \omega^{2} / 2\right)\left(u_{1}+u_{2}^{2}\right) / u_{0}^{2}$ and the Coulomb potential $V(\mathbf{u})=$ $-(\alpha / R)\left(u_{0} / \sqrt{u_{1}^{2}+u_{2}^{2}} \sqrt{16}\right.$, and we find that the identification $R^{2}=q$ for the coordinate systems (63) of all spectral properties of the two potentials remains valid. Therefore we can interpret the deformation parameter $q$ in the hyperbolic potentials as a curvature term.

\section{References}

[1] Arai, A.: Exactly Solvable Supersymmetric Quantum Mechanics. J. Math. Anal. Appl. 158 (1991) $63-79$.

[2] Böhm, M., Junker, G.: Path Integration Over Compact and Noncompact Rotation Groups. J. Math. Phys. 28 (1987) 1978-1994.

[3] Cho, S.: Quantum Mechanics on the h-deformed Quantum Plane. math-ph/9804015, J. Phys. A: Math. Gen. (1998), to appear.

[4] Duru, I.H.: Path Integrals Over SU(2) Manifold and Related Potentials. Phys. Rev. D 30 (1984) 2121-2127.

[5] Dutt R., Khare, A. and Varshni, Y. P.: "New Classes of Conditionally Exactly Solvable Potentials in Quantum Mechanics", J.Phys.A: Math.Gen. 28 (1995) L107-L113.

[6] Eğrifes, H., Demirhan, D., Büyükkıhç, F.: Polynomial Solutions of the Schrödinger Equation for the "Deformed" Hyperbolic Potentials by Nikiforov-Uvarov Method. Ege University preprint, August 1998. Physica Scripta, in press.

[7] Feynman, R.P., Hibbs, A.: Quantum Mechanics and Path Integrals. McGraw Hill, New York, 1965.

[8] Fischer, W., Leschke, H., Müller, P.: Path Integration in Quantum Physics by Changing the Drift of the Underlying Diffusion Process: Application of Legendre Processes. Ann. Phys. (N.Y.) 227 (1993) $206-221$.

[9] Gendenshteĭn, L.É.: Derivation of Exact Spectra of the Schrödinger Equation by Means of Supersymmetry. JETP Lett. 38 (1983) 356-359.

Gendenshteŭn, L.É., Krive, I.V.: Supersymmetry in Quantum Mechanics. Sov. Phys. Usp. 28 (1985) 645-666.

[10] Grosche, C.: Path Integral Solution of a Class of Potentials Related to the Pöschl-Teller Potential. J. Phys. A: Math. Gen. 22 (1989) 5073-5087.

[11] Grosche, C.: The Path Integral on the Poincaré Disc, the Poincaré Upper Half-Plane and on the Hyperbolic Strip. Fortschr. Phys. 38 (1990) 531-569.

[12] Grosche, C.: Path Integral Solution of Scarf-Like Potentials. Nuovo Cimento B 108 (1993) 13651376.

[13] Grosche, C.: Path Integration via Summation of Perturbation Expansions and Application to Totally Reflecting Boundaries and Potential Steps. Phys. Rev. Lett. 71 (1993) 1-4.

[14] Grosche, C.: Conditionally Solvable Path Integral Problems. J. Phys. A: Math. Gen. 28 (1995) 58895902. 
[15] Grosche, C.: Conditionally Solvable Path Integral Problems. II. Natanzon Potentials. J. Phys. A: Math. Gen. 29 (1996) 365-383.

[16] Grosche, C., Pogosyan, G.S., Sissakian, A.N.: Path-Integral Approach to Superintegrable Potentials on the Two-Dimensional Hyperboloid. Phys. Part. Nucl. 27 (1996) 244-278.

[17] Grosche, C., Steiner, F.: Handbook of Feynman Path Integrals. Springer Tracts in Modern Physics 145. Springer, Berlin, Heidelberg, 1998.

[18] Inomata, A., Kuratsuji, H., Gerry, C.C.: Path Integrals and Coherent States of SU(2) and SU(1,1). World Scientific, Singapore, 1992.

[19] Junker, G.: Supersymmetric Methods in Quantum and Statistical Physics. Springer, Berlin, Heidelberg, 1996.

[20] Kleinert, H.: Path Integrals in Quantum Mechanics, Statistics and Polymer Physics. World Scientific, Singapore, 1990.

[21] Kleinert, H., Mustapic, I.: Summing the Spectral Representations of Pöschl-Teller and Rosen-Morse Fixed-Energy Amplitudes. J. Math. Phys. 33 (1992) 643-662.

[22] Lemieux, A., Bose, A.K.: Construction de potentiels pour lesquels l'équation de Schrödinger est soluble. Ann. Inst. Henri Poincaré 10 (1969) 259-270.

[23] Lévai, G.: On Some Exactly Solvable Potentials Derived from Supersymmetric Quantum Mechanics. J. Phys. A: Math. Gen. 25 (1992) L521-L524.

[24] Олевский, М.Н.: Триортогональные системы в пространствах постоянной кривизны, в которых уравнение $\Delta_{2} u+\lambda u=0$ допускает полное разделение переменных. Mam. Сб. 27 (1950) 379-426.

[Olevskiı̌, M.N.: Triorthogonal Systems in Spaces of Constant Curvature in which the Equation $\Delta_{2} u+$ $\lambda u=0$ Allows the Complete Separation of Variables. Math. Sb. 27 (1950) 379-426 (in Russian)].

[25] Nag N., Roychoudhury, R. and Varshni, Y. P.: Conditionally Exactly Soluble Potentials and Supersymmetry. Phys. Rev. A 49 (1994) 5098-5099.

[26] Schulman, L.S.: Techniques and Applications of Path Integration. John Wiley \& Sons, New York, 1981. 